\title{
La figure de l'exécutif pendant les deux ministères Neufchâteau
}

Dominique Margairaz

\section{(2) OpenEdition \\ 1 Journals}

Édition électronique

URL : https://journals.openedition.org/ahrf/826

DOI : 10.4000/ahrf.826

ISSN : 1952-403X

\section{Éditeur :}

Armand Colin, Société des études robespierristes

\section{Édition imprimée}

Date de publication : 1 juin 2003

Pagination : 81-99

ISSN : 0003-4436

\section{Référence électronique}

Dominique Margairaz, «La figure de l'exécutif pendant les deux ministères Neufchâteau », Annales

historiques de la Révolution française [En ligne], 332 I avril-juin 2003, mis en ligne le 22 avril 2008, consulté le 24 avril 2022. URL : http://journals.openedition.org/ahrf/826 ; DOI : https://doi.org/ 10.4000/ahrf.826 


\title{
LA FIGURE DE L'EXÉCUTIF PENDANT LES DEUX MINISTÈRES NEUFCHÂTEAU
}

\author{
DOMINIQUE MARGAIRAZ
}

\begin{abstract}
Inscrite dans l'intervalle de deux brèves séquences $(28$ messidor-23 fructidor an V; 29 messidor an VI - 4 messidor an VII) l'expérience ministérielle de François de Neufchâteau à I'Intérieur présente des traits originaux dont les déterminations sont à rechercher à la fois dans le contexte institutionnel issu des compromis de l'an III et dans la position propre de l'intéressé, liée à son parcours singulier et à l'expérience accumulée au cours de celui-ci. Par l'analyse des modalités d'une pratique, on s'attache ici à cerner un style et à montrer comment le ministre, loin de se cantonner dans les bornes constitutionnelles assignées à sa fonction, a constamment tenté de l'investir d'une dimension politique d'initiative, tendant ainsi à revendiquer pour l'Exécutif ministériel une fonction d'initiative des lois.
\end{abstract}

Mots clés : Initiative des lois ; responsabilité ; François de Neufchâteau; Intérieur ; bureaucratie.

La figure de l'Exécutif pendant les deux ministères François de Neufchâteau se construit à la rencontre d'une expérience, d'un contexte et d'une pratique. L'expérience s'inscrit dans la longue durée d'une carrière administrative et politique, la première entamée à la fin des années soixante-dix du siècle, la seconde, amorcée dès l'année 1789 et impulsée de façon décisive en 1791 par l'élection du futur ministre à l'Assemblée législative. Le contexte idéologique et politique articule les données immédiates issues des options constitutionnelles de l'an III et celles qui résultent de la plus longue durée des débats et des pratiques qui ont marqué les destinées de l'Exécutif dès les commencements de la Révolution. La pratique ministérielle, enfin, est renfermée dans le temps bref des deux passages de François de Neufchâteau à l'Intérieur ( 28 messidor -23 fructidor an V; 29 messidor an VI - 4 messidor an VII).

De la figure de l'Exécutif qui se dessine pendant cet intervalle d'à peine quatorze mois, la mémoire historique a retenu deux aspects : l'un, positif, 
associe le ministre, sous les traits de l'administrateur énergique, à l'action résolue en faveur d'une exécution prompte des lois et du redressement, notamment économique, de la République; l'autre, négatif, l'associe sous les traits du manipulateur, à l'intervention illégale du Directoire dans le scrutin de l'an VII, en violation manifeste de la séparation des pouvoirs et de la liberté de suffrage (1). Si nous nous tournons vers les contemporains, nous découvrons approximativement la même image, assortie de nuances plus sombres : d'un côté, c'est l'administrateur volontariste que les députés des Cinq-Cents et des Anciens mettent en cause en prairial an VII, pour ses initiatives réformatrices jugées coûteuses et dont la régularité même est mise en doute; de l'autre, l'agent docile de la dictature directoriale, stigmatisé pour son intrusion dans le processus électoral de germinal (2). Des contemporains aux historiens, seule la tonalité du jugement porté sur l'œuvre accomplie change. De l'un à l'autre, il semble que l'histoire ait réalisé avec succès le pari engagé par François de Neufchâteau lorsqu'il prophétisait, en introduction du recueil de ses actes publics : «Le temps mettra tout à sa place » (3). Mais, qu'elle procède de la visée des contemporains ou de la focale rétrospective des historiens, l'image rassemble dans l'un et l'autre cas les deux traits, complémentaires, de l'Exécutif sous le Directoire : le premier incarne la dimension administrative de l'exécution des lois, le second la figure politique de l'Exécutif, assumant non seulement une fonction d'exécution, mais aussi une autorité, un véritable pouvoir reposant sur une délégation de souveraineté qui en fait un rival potentiel pour la représentation nationale.

À la lumière d'une exploration approfondie de la pratique ministérielle de l'intéressé d'une part, des développements récents de la réflexion sur le pouvoir exécutif pendant la Révolution et des révisions auxquelles ils font droit, de l'autre, il paraît intéressant de mettre en évidence, dans un numéro de la revue consacré à l'Exécutif, plusieurs traits qui singularisent l'approche de la fonction exécutive propre au ministre de l'Intérieur : celle-ci actualise en effet concrètement certaines des difficultés repérées et des aspirations exprimées lors des débats constitutionnels de l'an III ; elle se révèle par

(1) Cf. par exemple Jacques Godechot, Les institutions de la France sous la Révolution française et l'Empire, PUF, Paris, 1968, ou Denis WoRONOFF, La République bourgeoise de Thermidor à Brumaire 1794-1799, Nouvelle histoire de la France contemporaine, Paris, Seuil, 1972. Pour la figure noire : Marcel REINHARD, Le département de la Sanhe sous le régime directorial, Saint-Brieuc, Presses bretonnes; et plus récemment : Bernard GAINOT, 1799, Un nouveau jacobinisme? Paris, Éd. du CTHS, 2001.

(2) Les mises en causes de la gestion ministérielle de François de Neufchâteau aux Cinq-Cents et aux Anciens s'étalent sur les mois de germinal à thermidor an VII et se déploient sur deux fronts : la gestion des fonds et le soupçon d'irrégularités touchant notamment la fourniture des hospices; le rôle tenu dans les élections de l'an VII, par l'entremise de certains commissaires du Directoire, poussés à tenir des réunions de notabilités pour tenter d'infléchir les votes avant les assemblées primaires et les assemblées électorales.

(3) Recueil des lettres circulaires, instructions, programmes, discours et autres actes publics émanés du citoyen François (de Neufchâteau) pendant ses deux exercices du ministère de l'Intérieur, Paris, Imprimerie de la République, an VII-anVIII, 2 vol., (abrégé par la suite sous la forme Recueil...). 
ailleurs réellement anticipatrice, non seulement des ruptures consulaires, mais également des débats repris à nouveaux frais sous la Restauration (4).

Pour comprendre les enjeux engagés par cette pratique, il paraît nécessaire de rappeler les coordonnées institutionnelles qui la norment, à la lumière des compromis constitutionnels de l'an III, bien sûr, mais aussi des débats qui les ont précédés et des questions qui, loin d'être tranchées par ceux-ci, demeurent en suspens pendant toute la période directoriale. Car c'est précisément dans les interstices demeurés pour ainsi dire vides entre les articles laconiques de la Constitution que se dessinent les traits propres de la pratique ministérielle de François de Neufchâteau. Les Thermidoriens avaient-ils réellement « compris que l'histoire et la politique allaient suivre un chemin différent de celui tracé par leur créature constitutionnelle»? Sans juger ici de la réalité de la clairvoyance prêtée aux constituants par Paolo Colombo dans un article récent de la revue, force est de constater que la facture imprimée par François de Neufchâteau à ses deux exercices ministériels semble actualiser ce divorce (5).

L'attention récente accordée au statut et au rôle de l'Exécutif, particulièrement au moment de la tentative de stabilisation directoriale, a revalorisé l'ampleur des débats et des questions soulevées dans la phase préparatoire comme dans la phase active de l'élaboration du texte constitutionnel (6). Or, loin de s'éteindre avec le vote du texte en fructidor an III, l'intérêt porté à la question de la définition et de l'organisation de l'Exécutif demeure un pôle majeur de la réflexion jusqu'en l'an VIII, vivifié par l'actualité de la révision constitutionnelle. Mais en réalité, l'effort théorique de longue haleine pour penser la nature et la fonction gouvernementale, ainsi que les formes organiques qu'il convient de lui donner, s'amorce dès 1790-1791, à l'occasion des affrontements sur la question de l'irresponsabilité des ministres devant l'Assemblée, et, naturellement, lors des débats relatifs au veto royal. À cette époque, affirme Daunou en l'an III, toute l'énergie des constituants était tendue vers un but unique, «énerver la puissance exécutive » (7). Il serait néanmoins erroné de ramener à un enjeu politique purement conjoncturel la portée de la réflexion théorique qui s'esquisse alors. D'entrée de jeu fut posée la question, cruciale pour notre propos, de la nature propre de l'Exécutif, par le biais du couple volonté/action. «Apparemment, le schéma

(4) Voir notamment, La Constitution de l'an III ou l'ordre républicain, Dijon, Éditions de l'Université de Dijon, 1998. Sur François de Neufchâteau, Dominique MARGaIRAZ, Du lieu commun à l'esprit public. François de Neufchâteau. Essai de biographie intellectuelle. Thèse, Université de Paris I, 2001, 3 vol.

(5) Paolo Colombo, "La question du pouvoir exécutif dans l'évolution institutionnelle et le débat politique révolutionnaire $", A . H . R . F, 2000, \mathrm{n}^{\circ}$ 1, pp. 1-26.

(6) Sur le pouvoir exécutif, l'ouvrage de Joseph BARTHELEMY, Le rôle du pouvoir exécutif dans les républiques modemes, Paris, 1907, demeure une référence très utile permettant de situer le problème dans la longue durée. Pour les révisions récentes, outre les actes du colloque sur la Constitution de l'an III et l'article de Paolo Colombo, déjà cités, voir aussi Michel VERPEAUX, La naissance du pouvoir réglementaire 1789-1799, Paris, PUF, 1991.

(7) Daunou, le 5 thermidor an III, Moniteur, $n^{\circ}$ du 11 thermidor an III. 
constitutionnel de la division des pouvoirs... marque fortement la fracture entre les deux pouvoirs en question", affirme Paolo Colombo (8). Mais, parallèlement, il montre que les députés ne demeurèrent pas moins préoccupés de savoir comment éliminer la contradiction entre volonté et action, conscients que chaque fonction publique implique l'exercice d'une forme de volonté, ce qui revient à poser le problème de l'unité d'action, c'est-à-dire de la conformité de l' "agir » au «vouloir». En l'an III, les solutions théoriques et institutionnelles apportées à ce problème de l'unité d'action par le gouvernement révolutionnaire apparaissent rétrospectivement comme la cause directe de la Terreur, envisagée de ce point de vue comme pur produit de la confusion des pouvoirs. Néanmoins, comme le rappelle Michel Verpeaux, les conventionnels s'étaient préoccupés davantage que leurs précécesseurs de faire appliquer les décrets de la Convention, manifestant leur volonté de traduire concrètement en actes les décisions de l'Assemblée et de les faire triompher de toutes les résistances, juridiques et politiques (9). Cette leçonlà, du moins, demeurait bien vivante dans l'esprit des constituants de l'an III. Aussi est-ce dans la hantise d'un double héritage que ceux-ci abordent la question de l'Exécutif, avant tout soucieux d'élaborer une formule assurant l'équilibre des pouvoirs, ou, pour parler dans les termes alors en usage, la «balance» des pouvoirs.

Plusieurs auteurs s'accordent à relever le décalage observable entre la sécheresse, voire l'indétermination des articles constitutionnels relatifs à l'Exécutif en regard de la richesse et de l'acuité des débats poursuivis à l'époque, qui débordent largement les limites du cercle étroit de la Commission des Onze comme celles de l'enceinte de l'Assemblée (10). Décalage directement à l'origine de l'écart qui devait par la suite apparaître entre théorie constitutionnelle et pratique du pouvoir exécutif. Dans ses grandes lignes, le schéma constitutionnel est simple. Il atteste en premier lieu de la reconnaissance d'un pouvoir exécutif fort, propre à faire obstacle à toute tentation de retour à une tyrannie d'assemblée. L'Exécutif n'est pas seulement investi d'une fonction stricte d'exécution des lois, mais reconnu doué d'une volonté propre, d'une «pensée», dans laquelle s'incarne l'essence du gouvernement. "Le pouvoir exécutif [affirme Thibaudeau] consiste dans la pensée du gouvernement ». En théorie, cela revient à reproduire, au sein même de l'Exécutif cette fois, la distinction volonté/action. Formellement, cette distinction se traduit par l'opposition entre le Directoire et les ministres. Au premier seul est délégué le " pouvoir » exécutif, qu'il exerce collégialement, dans le cadre de ses délibérations, non seulement admises mais précisément codifiées. Aux seconds, strictement

(8) Paolo COLOMBO, art. op. cit, pp. 4-5.

(9) Michel VERPEAUX, op. cit.

(10) Notamment Michel VERPEAUX et Paolo COLOMBo, op. cit. 
subordonnés au Directoire qui les choisit et les révoque à son gré, sont dévolus les détails de l'exécution des lois. Ils font ainsi figure de simples exécutants de la politique décidée par le Directoire, de simple commis selon l'expression de Jacques Godechot, $d$ ' «agents généraux d'exécution » pour reprendre l'appellation sous laquelle ils sont significativement désignés dans les projets constitutionnels (11). Ne formant pas conseil, ils n'ont aucune vocation délibérative, et sont entièrement voués à des tâches d'exécution consistant, d'une part, à traduire les textes législatifs et les arrêtés du Directoire en un ensemble de dispositifs techniques et de tâches concrètes qui les rendent pratiquement applicables, d'autre part, à s'assurer de la prompte mise en œuvre de ceux-ci par les corps administratifs qui leur sont subordonnés, et par les tribunaux. Ces dispositions ont pour conséquence, entre autres, que les ministres n'ont pas vocation à appréhender l'ensemble des données politiques qui déterminent l'activité délibérative et décisionnelle du Directoire ou orientent l'action du législateur, mais seulement celles qui regardent leur propre département, au demeurant rigoureusement délimité par ce dernier (12).

Tel qu'il apparaît dans son laconisme, le texte de la Constitution dissimule la profondeur de la pensée constitutionnelle développée en l'an III. Plusieurs aspects de celle-ci, encore une fois d'importance pour le présent propos, méritent d'être relevés. Si l'acte constitutionnel est « fils de la question gouvernementale ", il n'en reste pas moins que le thème du gouvernement s'inscrit dans le cadre plus large de la recherche d'une nouvelle organisation des pouvoirs, qui rende possible la collaboration de ceux-ci sur la base de la séparation des fonctions (13). «On parle beaucoup de division des pouvoirs, affirme alors Mme de Staël dans une lettre à Roederer, et c'est peut-être leur union le problème le plus difficile " (14).

(11) Jacques GODECHOT, op. cit., p. 466.

(12) Le décret du 10 vendémiaire an IV fixe l'organisation et les attributions des différents ministères. Les attributions précises du ministre de l'Intérieur sont les suivantes : La correspondance avec les autorités administratives et les commissaires du Directoire exécutif auprès desdites autorités. Le maintien du régime constitutionnel et l'exécution des lois touchant les domaines suivants : les assemblées communales, primaires et électorales. Les prisons, maisons d'arrêt, de justice et de réclusion. Les hopitaux civils, les établissements et ateliers de charité, les établissement destinés aux sourds-muets et aux aveugles. La confection et l'entretien des routes, ponts, canaux et autres travaux publics. Les mines, minières et carrières. La navigation intérieure, le flottage, le halage. L'agriculture, les déssèchements et défrichements. Le commerce. L'industrie les arts et inventions, les fabriques, les manufactures les aciéries. Les primes et encouragements sur ces divers objets. La surveillance, la conservation et la distribution du produit des contributions en nature. L'instruction publique, les musées et autres collections nationales. Les poids et mesures. La formation des tableaux de population et d'économie politique, des produits territoriaux, des produits des pêches sur les côtes, des grandes pêches maritimes, et de la balance du commerce. L'exécution des lois relatives à la police générale, à la sûreté et la tranquillité de la République, la répression du vagabondage et de la mendicité, la Garde nationale sédentaire et le service de la gendarmerie sont transférés en nivôse an IV au ministère de la Police. Duvergier DE Hauranne, Collection des lois, décrets, ordonnances, réglements et avis du Conseil d'État 1788-1824, Paris, Guyot et Scribe, 1834 (2e édition), volume 9.

(13) Paolo CoLomBo, art. op. cit. pp. 13-14

(14) Id. 
Au-delà de la personnalité de la fille de Necker, très fidèle sur ce thème à la philosophie de son père, Paolo Colombo montre qu'il existe au même moment à l'Assemblée tout un groupe de théoriciens favorables à une division moins rigide des pouvoirs, représenté notamment par Ehrmann (15). De son côté, Sieyès, dont le projet complexe est finalement rejeté par ses collègues, plaide pour la création d'un pouvoir neutre, garant de la constitutionnalité des lois et chargé de résoudre les conflits entre l'Exécutif et les chambres (le «jury constitutionnaire»). Par ailleurs, sa conception du "gouvernement », qu'il distingue soigneusement de ceux qu'il désigne comme les "ordonnateurs de l'action de la loi », le conduit à imaginer un corps de représentants de sept membres, ayant pour mission de "veiller aux besoins du peuple et à ceux de l'exécution de la loi, et de proposer à la législation toute loi, règlement ou mesure qu'il jugera utile». En d'autres termes, il conçoit d'accorder à l'Exécutif l'initiative des lois. «Pour faire la loi, renchérit Mme de Staël, il faut préalablement connaître les besoins de l'administration et non les découvrir au moment de la promulgation ou de l'application... Les connaissances que le pouvoir exécutif peut seul réunir sont nécessaires à la confection de la loi " (16). À la différence de Sieyès, c'est dans la fonction ministérielle qu'elle repère les compétences propres à investir celle-ci d'une prérogative d'initiative des lois. «Dans l'Assemblée constituante, observe-t-elle, il est arrivé vingt fois qu'on décrétait en Finances, à la Guerre, etc. une chose tout à fait impossible et qu'une simple objection d'un ministre aurait absolument empêché » (17). En l'an III, le dispositif constitutionnel rend à ce point étanche la séparation des pouvoirs que le Directoire et les ministres, rigoureusement exclus de l'enceinte de l'Assemblée, ne peuvent en aucune façon interférer dans les débats et peuvent seulement prier le corps législatif de prendre en considération telle question par le biais d'un message. Sauf requête expresse des députés, les ministres ne peuvent pas davantage éclairer de leur expérience et des données qu'ils ont en leur possession les décisions des assemblées.

Ainsi, en marge du courant majoritaire favorable à une stricte séparation des pouvoirs excluant la participation de l'Exécutif à la production de normes juridiques générales, que ce soit sous la forme d'un partage de l'initiative des lois ou sous celle de l'exercice d'un pouvoir réglementaire, il existait bel et bien, sinon un courant cohérent et clairement structuré, du moins une sensibilité politique hostile à une étanchéité trop rigide des différents pouvoirs et des organes qui les représentent. Cette division était, dans cette optique, de nature à compromettre le processus d'élaboration éclairé de la

(15) Id., pp. 16-17.

(16) Germaine DE STAËL, Réflexions sur la paix intérieure, Paris, 1795, cité par Lucien JAUME «L'esprit de Coppet et l'organisation du pouvoir exécutif » dans La Constitution de l'an III ou l'ordre républicain, Éditions de l'Université de Dijon, 1998.

(17) Lettre à P. L. Roederer, 21 frimaire an III, cité par Lucien JAUME, art. op. cit. 
loi comme celui de sa mise en application, une loi jugée insuffisante ou mauvaise étant d'autant plus difficile à mettre en œuvre par ceux qui en sont chargés. Les constituants s'en remettaient finalement aux vertus d'un ressèrement des liens hiérarchiques subordonnant les corps administratifs aux ministres, et à celles du contrôle exercé par les commissaires du Directoire, pour obtenir la prompte et stricte application de la loi. C'était parier beaucoup sur l'efficacité de la bureaucratie ministérielle et, paradoxalement, beaucoup attendre d'une fonction par ailleurs durablement suspecte à leurs yeux, toujours environnée du spectre de la «tyrannie ministérielle».

Nommé juge au Tribunal de cassation par décret de la Convention le 11 nivôse an III et installé dans ses fonctions en avril 1795, François de Neufchâteau fut certainement un observateur attentif des travaux d'élaboration du texte constitutionnel. Député des Vosges sous la Législative où il siège au comité de Législation, nombre de conventionnels sont connus de lui. Grand lecteur de journaux et de périodiques, il se tient à coup sûr au courant du débat public, auquel il ne semble pourtant pas tenté de mêler sa voix : on ne repère aucun texte de sa main parmi les projets adressés à la Commission des Onze (18). Il serait en réalité hasardeux de prétendre le situer dans la diversité des courants qui s'expriment alors. Nous ne possédons en effet aucun témoignage de l'état d'esprit qui anime alors le futur ministre, à l'exception d'une lettre d'encouragement, d'un contenu toutefois assez vague, adressée à Sieyès le 18 messidor an III, antérieure donc à l'exposé précis par celui-ci de son projet constitutionnel devant la Convention (19). Du côté de «l'esprit de Coppet », rien de tangible non plus. S'il fut, dans l'Ancien Régime finissant, lecteur et admirateur de Necker dont il a médité le traité De l'administration des finances de la France, aucun élément ne permet d'établir qu'il eut accès aux textes postérieurs de l'ancien directeur général des Finances, qui auraient pu orienter sa propre réflexion, en particulier le commentaire de la Constitution de 1791 que celui-ci fait paraître en 1792, Du pouvoir exécutif dans les grands États. Le titre n'apparait pas au catalogue de sa bibliothèque, pas plus, d'ailleurs, que De la Révolution française, publié en 1796. Aucune trace non plus des Réflexions sur la paix intérieure de Germaine de Staël, ni de l'opuscule paru en 1798, Des circonstances actuelles qui peuvent terminer la Révolution et des principes qui doivent fonder la République en France. On ne dispose donc d'aucune profession de foi constitutionnelle du futur ministre et, en la circonstance, son étonnant commentaire de la Constitution de 1791, L'origine ancienne des principes modernes, ne peut en aucun cas en tenir lieu

(18) Les papiers de la Commission des Onze, A.N., C 226-C 232. Sur les textes adressés à la Commission, cf. Françoise BRUNEL, "Aux origines d'un parti de l'ordre : les propositions de constitution de l'an III ", dans Mouvements populaires et conscience sociale, XV7e-XIXe siècles, Paris, Maloine, 1985.

(19) Lettre de François de Neufchâteau à Sieyès, 18 messidor an III, A.N., 284 AP 9, dossier 5. 
ni ne permet en aucune façon de dégager par anticipation une philosophie tranchée. Au demeurant, François de Neufchâteau n'est pas un théoricien. Il appartient à cette catégorie d'administrateurs, hommes d'action pour lesquels la réflexion passe par une méditation sur sa propre expérience, dont elle reste, sur le fond comme dans la forme, étroitement solidaire et dépendante. Aussi l'analyse de ses pratiques de travail, replacées dans la trame d'un itinéraire complexe, s'avère-t-elle beaucoup plus révélatrice de sa conception gouvernementale que d'hypothétiques conjectures en termes d'« influence», concept mou de faible ou nulle valeur heuristique.

Cet itinéraire professionnel, entamé dans les années 1770, lui a en effet permis d'expérimenter toutes les positions virtuellement distribuées dans l'éventail des fonctions afférentes au pouvoir d'État, fonction législative, exécutive ou judiciaire. Dans l'Ancien Régime, François de Neufchâteau a connu et éprouvé concrètement la réalité de l'exercice d'un pouvoir administratif encore mal dégagé du judiciaire en qualité de lieutenant civil et criminel au présidial de Mirecourt (1778-1783), de subdélégué de l'intendant de Lorraine (1781-1783) et de procureur du roi au Conseil supérieur du Cap Français (1783-1787). Pendant la Révolution, il remplit, simultanément ou en alternance, les fonctions de juge de paix du canton de Vicheray, d'administrateur du département des Vosges (1790-1791 puis 1792-1793), de député à la Législative, de juge au Tribunal de cassation, de commissaire du Directoire exécutif près l'administration centrale des Vosges (1795-1796), de ministre, de directeur, puis de ministre à nouveau. Signalons d'entrée la singularité de son destin ministériel, contitutionnellement voué à l'«agir» dans le cadre de la réalisation de tâches administratives, mais encadré par deux séquences - son commissariat dans les Vosges et son élection au sein du Directoire - placées sous le signe du « vouloir » et, pour la deuxième, de la participation directe au gouvernement. Un tel parcours, accompli dans des conditions et des contextes, politique et matériel, souvent délicats, l'avait mis dans l'obligation de s'interroger, non de façon abstraite, mais en réaction aux difficultés rencontrées dans l'accomplissement de ses diverses missions, sur les conditions d'exercice de ses différentes fonctions et sur les modalités d'une nécessaire articulation entre les pouvoirs. S'il n'a jamais rassemblé en un corps de propositions théoriques le fruit de cette expérience, l'idéal de service qui l'anime, intimement lié à ses chances de survie dans la sphère publique, le conduit plus d'une fois, dans le cadre des relations hiérarchiques qu'il entretient avec supérieurs et subordonnés, à exprimer constats et jugements qui font apparaître les repères qui organisent sa conception d'un gouvernement efficace. Sa pratique ministérielle même, dans l'écart qu'elle creuse avec la norme constitutionnelle, manifeste la haute opinion qu'il se fait de la fonction, et, au-delà de l'accomplissement zélé des tâches d'exécution proprement dites, la perception d'une articulation possible entre fonction d'exécution et fonction d'initiative, fondée sur la responsabilité. C'est à cette pratique qu'il faut s'attacher mainte- 
nant, afin d'en mettre en évidence quelques traits saillants, qui façonnent une figure originale de l'Exécutif. Les différentes déclinaisons de celle-ci sont étroitement liées entre elles : c'est en effet dans la rigueur de la mise en œuvre des tâches strictes d'exécution qui lui sont assignées que le ministre puise la substance d'une interprétation extensive de sa fonction.

La figure de l'Exécutif ministériel apparaît comme la résultante d'une gamme étentue d'interventions qui ne relèvent pas de la même dynamique, mais s'inscrivent dans une indiscutable continuité logique et des séquences temporelles identifiables. Au fondement de sa pratique, une exceptionnelle activité déployée pour parvenir à une diligente et stricte application des lois, objectif qui requiert à son tour une série d'opérations et de médiations de nature différente. Les premières ont pour objet la traduction des lois, en un double sens, pédagogique et technique; les secondes le contrôle de la mise en œuvre effective, de la promptitude et de la régularité des actes administratifs (information des administrations subalternes et des citoyens ; dispositifs opérationnels) qui rendent possibles le respect de la loi par la généralité ou telle catégorie de citoyens.

On entend par traduction pédagogique l'explicitation de la loi, de ses attendus et de sa portée, pour une meilleure intelligibilité de celle-ci par les corps administratifs, eux-mêmes chargés d'en organiser l'exécution par les administrations de canton qui leur sont subordonnées. Il s'agit donc de s'assurer d'une bonne compréhension des termes de la loi par un personnel élu dont les compétences juridiques, administratives et même linguistiques ou arithmétiques sont fréquemment mises en doute ou prises en défaut. Certains textes, assortis d'un dispositif technique sophistiqué, requièrent un important investissement pédagogique de la part du ministre, qui se traduit par des envois réitérés de circulaires destinées chaque fois à préciser les attentes du législateur C'est le cas par exemple de la circulaire du 5 vendémiaire an VII consacrée à l'explicitation de la loi du 24 frimaire sur la liquidation de la dette des hospices et à la clarification des modalités financières qu'elle prévoit (20). Dans la même catégorie peut être rangée la circulaire du 21 frimaire an VII relative à la loi du 11 frimaire précédent règlant la liquidation des dépenses arrièrées des administrations : «Pour vous rendre cette exécution plus facile [commente le ministre] je vais entrer dans quelques explications qui vous en feront saisir l'ensemble »; ou celle du 30 germinal an VII relative à l'exécution du tableau des recettes et dépenses municipales des cantons composés de plusieurs communes : « Je désire vous entretenir de l'exécution de quelques articles des deux lois du 11 frimaire qui ne me paraissent pas avoir été assez entendus par plusieurs administrations... » (21).

(20) La circulaire du 5 vendémiaire figure au tome I, pp. 186-192.

(21) Recueil..., respectivement tome I, pp. 327-335 et tome II pp. 171-176. 
Par traduction «technique ", il faut entendre la traduction des termes abstraits et généraux de la loi ou des arrêtés du Directoire, eux-mêmes réputés strictement conformes à l'esprit et la lettre de la loi, en un ensemble de dispositions concrètes qui les rendent applicables, de façon universelle, uniforme, sur l'ensemble du territoire: Ces dispositions associent les objectifs ou les impératifs définis par les textes normatifs à des opérations et des tâches précises assignées aux corps administratifs. L'arrêté du Directoire du 19 ventôse an VI relatif à la navigation intérieure, prescrivant qu'aucune usine, écluse, moulin, etc., ne pourra être établi sur une rivière navigable sans autorisation de l'administration centrale qui ne la donnera que sur autorisation expresse du Directoire présente ainsi des difficultés d'application en raison de l'indétermination des procédures à suivre. Les demandes, variées dans leur forme, sont souvent imprécises et incomplètes, le vou des administrations insuffisamment argumenté et pas assez clairement exprimé. «J'ai pensé qu'il était à propos de fixer une marche simple et régulière qui, en remplissant l'objet de l'arrêté, pût facilement être connue des administrés et suivie par les corps administratifs » expose le ministre dans son instruction du 19 thermidor an VI, avant de développer par le menu les dispositions précises qu'il a conçues "pour établir l'ordre et l'uniformité " (22). De son côté, la loi du 3 nivôse an VI établissant une taxe d'entretien des routes figure sans doute parmi celles qui requiéraient l'élaboration d'un dispositif des plus précis pour espérer une mise en place rationnelle et rapide des bureaux de perception. La détermination de l'emplacement optimal de chacun d'eux exigeait en particulier des compétences techniques, mais aussi des talents de négociateurs de la part des administrateurs amenés à justifier auprès de leurs concitoyens le bien-fondé de leur choix. La circulaire du 25 thermidor an VI a précisément pour objet de fixer une procédure uniforme pour l'ensemble des départements, et s'attache à décomposer en une série de tâches simples les étapes qui doivent conduire à la configuration d'un semis suffisamment dense pour permettre de capter l'ensemble des trafics, sans alourdir excessivement le coût de perception de la taxe (23). Bien des circulaires s'apparentent ainsi à de véritables circulaires d'application, sans lesquelles le respect de la loi demeurerait lettre morte. La circulaire du 11 frimaire an VII fixe ainsi les modalités administratives d'application de la loi contre les abus des entrepreneurs de spectacles, sans lesquelles la garantie de protection de la propriété littéraire serait abandonnée aux seuls recours contentieux. L'énoncé de règles contraignantes pour les entrepreneurs, sous la garantie des administrations municipales, procure le cadre réglementaire propice à la réalisation pleine et entière de l'action de la loi (24).

(22) Id, tome I, pp. 48-52.

(23) Id., pp. 53-63, citation p. 57.

(24) Id., pp. 311-315 
Ce volet normatif de l'activité du ministre resterait cependant d'une portée incertaine en l'absence d'un suivi et d'un contrôle sévère de l'exécution fidèle de ses prescriptions. Ce suivi requiert à son tour la tenue d'une correspondance régulière avec le ministre, soit de la part des administrations centrales sommées d'accuser ponctuellement réception de ses circulaires et de l'informer des dispositions prises par elles pour se conformer aux instructions qu'elles renferment et répercuter celles-ci au niveau local, soit de la part des commissaires du Directoire, dont les rapports, décadaires puis mensuels, mais aussi la correspondance courante, doivent le mettre à même d'apprécier les progrès de l'application des lois dans les départements et le degré de réactivité des corps administratifs. La correspondance avec les départements apparaît manifestement comme l'un des pivots de la stratégie ministérielle, condition de l'amélioration de l'efficacité administrative; si l'on s'en rapporte au nombre de circulaires ayant pour objet le rappel à l'ordre sur ce point des corps administratifs et des commissaires du Directoire, ainsi qu'aux menaces de sanctions dont il assortit ses admonestations. Au niveau central, il manifeste la même insistance sur la nécessité d'un traitement rapide de la correspondance, qu'il s'agisse de la distribution du courrier reçu entre les différents bureaux, de la rédaction d'extraits à usage du ministre, du classement, de l'archivage, ou, naturellement, de la préparation et de l'expédition des réponses (25). De fait, on observe une amélioration indiscutable du suivi départemental : progrès de la régularité de l'envoi de leurs comptes par les commissaires centraux, amélioration des taux de réponse des départements consécutive à l'émission de relances (26).

La correspondance entretenue avec les départements n'est pas moins importantes au regard de l'usage auquel elle est destinée : l'information récoltée se trouve en effet au fondement d'une évaluation rationnelle des difficultés rencontrées dans l'application des lois et d'une analyse des causes de celles-ci, ouvrant la possibilité d'une remédiation appropriée. C'est en effet l'appréciation quantitative et qualitative des performances des administrations qui détermine aux yeux du ministre la conduite à tenir en vue d'une amélioration des résultats : investissement pédagogique ou rhétorique accru, assorti, le cas échéant, de menaces disciplinaires afin de stimuler le zèle des corps administratifs, remédiation par la voie d'un arrêté du Directoire, ou par voie législative, sollicitée par l'envoi d'un message adressé, sur rapport du ministre, par le Directoire exécutif au Conseil des Cinq-Cents. Ce flot d'informations régulier est encore abondé par le résultat des enquêtes secto-

(25) Cf. sur ce point l'Ordre de travail des bureaux du ministre de l'Intérieur, 2 thermidor an VI, Recueil..., tome I, pp. 1-11.

(26) Sur l'évolution des envois des comptes des commissaires, cf. Lauriane ALBRECHT, « La correspondance administrative des autorités départementales avec le ministère de l'Intérieur sous le Directoire. Les comptes des commissaires et des administrations ", DEA, IHES-Paris I, 1999. 
rielles ou générales lancées par le ministre, qui ont pour objet de lui fournir la matière d'un tableau de la République, constamment actualisé et tenu à la disposition du Directoire ou, via celui-ci, du législateur, qui peuvent l'un comme l'autre à tout moment lui en réclamer les éléments. Questionnaires et tableaux sont ainsi régulièrement adressés aux corps administratifs avec la mission d'assurer la collecte des renseignements qui leur sont demandés auprès des administrations de canton. Attentif à la qualité comme à la promptitude des réponses, le ministre dresse le tableau d'honneur des départements les plus zélés, et tire argument des résultats obtenus pour exhorter les plus négligents à satisfaire sans délais à ses demandes : l'impulsion l'emporte alors sur la dimension technique de l'exécution, et la rhétorique persuasive du ministre manifeste la volonté, qu'il espère communicative, sans laquelle il n'est pas d'action possible (27). Les données issues des diverses enquêtes ou tableaux synthétiques adressés aux corps administratifs ou aux commissaires forment en effet à ses yeux la base d'une évaluation périodique des progrès de l'ordre républicain, témoignant de l'efficacité de l'investissement législatif et gouvernemental. Ainsi, dans l'accomplissement même des tâches qui lui sont constitutionnellement dévolues et le suivi rigoureux des départements, il puise la matière d'une réflexion de large ampleur, dont le volet prospectif ou les prolongements décisionnels lui échappent, mais auquel il prête néanmoins largement la main.

François de Neufchâteau se sent indiscutablement à l'étroit dans ses habits de ministre, taillés étriqués par la Constitution. Intégré dans l'appareil exécutif dès l'an IV en qualité de modeste commissaire du Directoire, il en a d'emblée expérimenté, quoique dans une position subordonnée, la face politique, celle de l'impulsion et du vouloir : les commissaires n'ont en effet aucune vocation à prendre part directement à l'administration des départements (il n'ont en particulier pas voix délibérative aux séances auxquelles ils sont admis), mais requièrent et surveillent l'exécution des lois et des arrêtés du Directoire (28). Ils sont donc à la fois l'œil et la voix de celui-ci dans les départements, rappelant constamment les administrateurs à leurs devoirs et

(27) Par exemple : tableau nominatif des départements ayant répercuté les directives du ministre sur les plantations, circulaire du 25 vendémiaire an VII, Recueil ..., tome I, pp. 232-242.

(28) Il n'existe aucune étude d'ensemble consacrée aux commissaires du Directoire. En attendant les résultats de l'enquête prosopographique lancée par Bernard Gainot dans le cadre de l'IHRF, on aura recours aux ouvrages généraux sur le Directoire, ou à des études de cas menées dans le cadre départemental. Notamment : Jean-René SURATTEAU, «Un commissaire du Directoire : François-Augustin Roussel », A.H.R.F., oct-déc. 1957, pp. 316-339; du même, Le département du Mont-Terrible sous le régime du Directoire, Paris, 1964; Marcel ReINHARD, Le département de la Sarthe sous le régime directorial, op. cit.; Pierte Clémendot, Le département de la Meurthe à l'époque du Directoire, Nancy, 1966; Jean Brelot, la vie politique en Côte d'Or sous le Directoire, Dijon, 1932. Plus récemment, Philippe Bourdin, Des lieux, des mots, des révolutionnaires. Le Puy de Dôme entre 1789 et 1799, Clermont-Ferrand, Institut d'études du Massif Central, 1995. En outre, sur les comptes des commissaires du Directoire : Albert MATHIEZ, «Les comptes décadaires des autorités du gouvernement révolutionnaire et des commissaires du Directoire ", R.H.M.C., tome IV, 1902, pp. 155-169. 
à leurs tâches, véritables "procurateurs d'exécution" dans les provinces. Lépisode lui a en outre procuré l'occasion de mesurer la distance qui sépare la vision parisienne des réalités de terrain, l'irréalisme de certaines décisions. Il en retire la conviction qu'il est nécessaire de faire connaître dans le plus grand détail l'état réel des départements, afin d'éclairer efficacement l'action du gouvernement et les décisions du législateur. C'est bien pourquoi son premier ministère est marqué par une sévère reprise en main des corps administratifs et des commissaires en ce sens - le contexte de fructidor s'y prêtait - et par le lancement d'une série d'enquêtes destinées à faire le point exact sur la situation de l'industrie, de l'instruction publique, des hôpitaux et des prisons, des finances communales... (29). En prairial an VI cependant, ce n'est plus seulement un obscur commissaire du Directoire exécutif qui accède pour la seconde fois au ministère de l'Intérieur, mais un directeur sorti de charge deux mois auparavant, qui s'est frotté pendant huit mois aux réalités de la grande politique, intérieure et extérieure, et a pénétré le mystère de tous les secrets de l'État. Durant cette brève période, en qualité de membre de l'Exécutif collégial, il s'est trouvé dépositaire de ce pouvoir d'impulsion et de décision par lequel se manifeste l'essence du vouloir gouvernemental. Une des particularités de la pratique ministérielle de François de Neufchâteau se révèle précisément dans la confusion permanente de l'agir et du vouloir que la Constitution avait entendue séparer artificiellement par la distinction hiérarchisée des fonctions directoriales et ministérielles.

Cette confusion se laisse en premier lieu repérer dans les initiatives développées en direction des lois demeurées inexécutées, faute justement d'impulsion clairement exprimée en ce sens et de détermination précise des modalités réglementaires d'application de celles-ci. C'est par exemple sur le rapport du ministre et sur la base du projet précis présenté par celui-ci au Directoire qu'est promulgué l'arrêté du 27 brumaire an VII organisant la mise en place de bureaux de poids publics dans les communes de plus de 5000 habitants, dont l'objet est de créer les cadres concrets de mise en application de l'article XXI de la loi du 14 mars 1790 confiant aux municipalités le soin de pourvoir à un service de pesage et mesurage public sur les halles et marchés urbains (30). Au-delà, il s'agit bien sûr de favoriser l'application de la loi du 1er vendémiaire an IV qui rend obligatoire l'usage des mesures républicaines, dont François de Neufchâteau s'efforce par ailleurs de faire progresser la diffusion. En second lieu, cette confusion ressort de la proprension du ministre à s'affirmer comme force de proposi-

(29) On ne compte pas moins de neuf circulaires disciplinaires mettant en particulier l'accent sur le thème de la correspondance, du 20 thermidor au 21 fructidor an $\mathrm{V}$.

(30) Arrêté du 27 brumaire an VII, Recueil..., Table chronologique, p. 358. Le rapport est résumé dans une lettre du ministre au chef de la quatrième division du ministère, A.N., F12 215. Circulaire du 14 floréal an VII, ibidem, pp. 190-193. 
tion et à s'immiscer dans l'activité règlementaire du Directoire, qui tend elle-même à s'émanciper des bornes constitutionnelles dans lesquelles elle se trouve renfermée. On relève ainsi un nombre élevé d'arrêtés dont le recueil de ses actes publics, qui les retient significativement comme lui étant imputables, ne rend qu'un faible compte en ne comptabilisant que ceux qui furent approuvés et promulgués par le Directoire. Les feuilles de travail du ministère de l'Intérieur font état d'un plus grand nombre de propositions, preuve qu'à défaut de l'avoir constamment inspirée, le ministre s'est efforcé de peser sur les orientations de la politique gouvernementale, au moins dans les domaines qui regardent son département. De la même façon, certaines proclamations, acte qui relève de la seule prérogative du Directoire, furent en réalité rédigées par lui (31). Enfin, sous la figure, revendiquée comme telle, $d$ ' « initiatives » en direction des divers domaines placés sous sa tutelle dans le cadre du «maintien du régime constitutionnel», on repère l'expression d'une véritable politique de mobilisation des corps administratifs, et, par leur intermédiaire, des citoyens, appelés à concourir à l'œuvre de redressement économique dont les orientations épousent les grandes lignes du discours officiel de l'encouragement, mais dont les options particulières et les modalités incitatives lui reviennent en propre. Si les initiatives en direction de l'industrie accompagnent indiscutablement l'infléchissement de la politique directoriale dans le sens d'un nationalisme économique de plus en plus affirmé, d'autres, comme la série des circulaires relatives au reboisement, dessinent un véritable programme de relèvement de l'agriculture et expriment une vision normative personnelle de l'action publique, en dehors de toute référence à un cadre législatif existant, anticipant au contraire l'investissement prescriptif que le législateur devrait être selon lui disposé à consentir. L'examen de cette séquence mérite qu'on s'y arrête, car elle offre au demeurant un exemple éclairant des enchaînements logiques et temporels qui organisent la démarche ministérielle. Comme c'est souvent le cas, celle-ci associe la sensibilité de l'administrateur des Lumières, l'expérience du commissaire du Directoire, et les constats ou les jugements du ministre sur la situation présente.

La prise de conscience réaliste de l'ampleur de la déforestation et des problèmes qu'elle induit à l'égard de la satisfaction des besoins, tant industriels que domestiques, remonte en effet à la période de son commissariat dans les Vosges. Dès cette époque, dans un véritable réquisitoire qui semble renvoyer les décideurs à leur propre incurie, il dresse à l'intention du ministre de la Police le constat de l'impossibilité de réprimer efficacement les délits forestiers, tant en raison de l'incertitude législative régnante, impu-

(31) Proclamation du 23 fructidor an $\mathrm{V}$ sur le rétablissement de la morale républicaine; proclamation du 23 pluviôse an VII sur les élections. Sur les circonstances de cette rédaction, cf. Recueil..., Table chronologique, pp. 334 et 380. 
table à l'absence de code forestier, qu'en raison du défaut d'agents compétents. Mal ou pas du tout payés, les gardes abandonnent leur poste, ceux qui demeurent sont corruptibles ou, le plus souvent, inaptes à remplir leur mission, faute d'instruction. À leur usage, le commissaire entreprend de rédiger un manuel renfermant notamment des formules types prêtes à l'emploi, et dont il envisage d'emblée la large diffusion sur l'ensemble du territoire (32). Les premières initiatives amorcées dès son arrivée au ministère en fructidor an V s'inscrivent dans la continuité de cette expérience. En l'absence de tout point d'appui législatif, deux circulaires du 22 fructidor an $\mathrm{V}$ entreprennent, via les corps administratifs, la mobilisation des propriétaires en faveur d'opérations de reboisement dont il fait valoir les perspectives hautement rémunératrices, parallèlement à la vertu civique dont la conjoncture les pare (33). L'intervention dans le cadre des moyens limités accordés à son ministère pour les «encouragements à l'agriculture » n'est cependant à ses yeux qu'un palliatif, pierre d'attente d'un programme ambitieux dont il expose très ouvertement en vendémiaire an VII, à l'intention des administrations centrales, ce que devraient être à ses yeux les grandes lignes (34). Sur la base d'un tableau exact du recul des surfaces boisées et des causes de celui-ci, c'est au législateur qu'il revient, non seulement de publier sans tarder un code forestier et de pourvoir aux moyens de le faire respecter, mais encore d'imaginer une politique rationnelle et efficace de reboisement, propre à préserver l'intérêt général des méfaits du seul jeu des intérêts particuliers et de la recherche du profit à court terme : «L'ignorance, la négligence et l'imprudence coupable de la part des propriétaires doivent être l'objet d'une attention sérieuse de la part des législateurs et du gouvernement [affirme sans détour le ministre]. J'ai lieu d'espérer [poursuit-il] que bientôt nos représentants vont enfin s'occuper des forêts. Je suis sûr qu'ils verront en grand le système des bois en France et qu'ils sauront déterminer, d'une manière fixe, la proportion à laquelle les forêts et plantations doivent être portées pour que les bois soient en rapport avec le reste des produits de la totalité du sol. La science de ces rapports est une des grandes données de l'économie politique. L'homme d'Etat doit calculer combien de champs en culture exigent de prairies; combien il devrait y avoir de têtes de bétail pour chaque hectare de terrain, et combien de grands végétaux, arbres fruitiers et forestiers, on doit avoir sur un domaine dans la proportion des avantages qu'ils procurent et de l'espace qu'ils exigent » (35).

(32) Lettres du 29 pluviôse et du 7 floréal an IV, Correspondance du commissaire du Directoire exécutif, A.D. Vosges, registres L 352 et 353.

(33) Circulaire du 22 fructidor an $V$ et programme des encouragements accordés aux citoyens qui feront des plantations, Recueil..., tome I, pp. LXXXI-LXXXV et LXXXVI-XC.

(34) Circulaire du 25 vendémiaire an VII, id., pp. 232-242.

(35) Id., pp. 236-237. 
C'est en réalité dessiner les orientations d'un vaste programme interventionniste, fondé sur les données de l'expertise scientifique et sur celles, empiriques, que sont à même de produire les bureaux de son ministère - bureaux de la quatrième division et bureau consultatif d'agriculture composé d'agronomes - suggérant, dans l'intérêt supérieur de la nation, un subtil dosage d'incitation et de contrainte.

De l'administration à la politique, de l'application des lois à la vision d'une politique agricole rationnelle et respectueuse de l'équilibre des ressources et des sols, le trajet est déterminé par l'expérience et la conscience qu'elle inspire d'une nécessaire articulation entre le travail des bureaux, appuyé sur la connaissance des réalités du terrain, et le processus décisionnel, qu'il relève de l'action gouvernementale ou de la production législative. En la circonstance cependant, aucun acte concret ne préfigure ou ne cherche à provoquer le prolongement concret de cet exposé programmatique. D'autres exemples permettent de le saisir. Ils mettent en évidence la manière dont, loin de s'en tenir à des tâches administratives de traduction du contenu politique des lois en un ensemble de dispositifs techniques, le ministre a réciproquement constamment cherché à suggérer la traduction politique des conclusions auxquelles le conduisaient le rassemblement et le traitement des données qu'il s'appliquait à réunir sans relâche.

Les messages du Directoire exécutif, assortis des rapports du ministre, sont le vecteur de cette interpellation du législateur. Significativement, François de Neufchâteau n'a nullement cherché à s'effacer derrière la personnalité collective de l'Exécutif directorial et revendique au contraire hautement l'initiative et la paternité de ces messages, dont la lettre, ou quelquefois le canevas seulement, sont élaborés dans ses bureaux. Le recueil de ses actes publics les comptabilise avec soin. Encore le décompte sous-estime-t-il encore une fois la dynamique dont les feuilles de travail du ministère révèlent toute la vigueur, faisant état d'un bien plus grand nombre de propositions. Au total, pas moins de 12 messages furent adressés à son initiative au Corps législatif pendant les deux mois de son premier ministère, 29 pendant.les douze mois de son second ministère. Ils couvrent à peu près l'éventail complet des secteurs placés sous sa surveillance par le décret du 10 vendémiaire an IV, mais leur répartition dans le temps n'est pas fortuite. Deux cas de figure peuvent se présenter : les uns s'inscrivent dans la dynamique du travail parlementaire, les autres procèdent d'une dynamique interne, et visent au contraire à amorcer l'activité du législateur, en proposant des mesures inspirées par les réalités de la gestion ministérielle, mais dont la nature requiert l'intervention législative. À cette configuration appartient par exemple le message du 11 fructidor an $\mathrm{V}$, accompagné d'un rapport circonstancié du ministre, proposant la nationalisation des droits de 
passage, bacs et bateaux des rivières navigables et canaux de la République (36). La démarche, motivée par l'urgence de trouver des financements pour assurer la remise en état des voies navigables, se trouve directement à l'origine du vote de la loi du 6 frimaire an VII, qui décrète la confiscation des droits de passage et prévoit transitoirement leur mise en régie, avant la mise en ferme ultérieure. La plupart du temps cependant, les impulsions ministérielles sont déterminées par la chronologie des débats, ou de l'absence de débats parlementaires. Le ministre en suit apparemment attentivement l'évolution, guettant l'occasion de s'inviter dans les délibérations des Cinq-Cents, ou préoccupé d'en hâter la conclusion aux Anciens. C'est ainsi par exemple qu'il tente d'appuyer l'idée d'une réforme de la loi sur l'instruction publique dont il dessine les contours en l'an VII, ou d'argumenter en faveur d'une réduction du nombre des cantons, en faisant opportunément passer messages et rapports lorsque ces thèmes viennent à l'ordre du jour (37). Il procède de la même façon pour faire connaître son engagement et les arguments qui jouent selon lui en faveur de l'établissement de la taxe sur la navigation intérieure, qui ne sera, de fait, instaurée que sous le Consulat avec le vote de la loi du 30 floréal an X. Dès le 29 pluviôse an VII, le ministre a saisi l'occasion offerte par la présentation aux Cinq-Cents du rapport de Borel-Vernière au nom de la commission fluviale, et le renvoi à ladite commission, pour faire parvenir un message assorti d'un rapport argumentant la nécessité de recourir à la taxe pour subvenir à l'entretien des rivières et canaux (38). Par un nouveau message du 12 ventôse, le Directoire, invoquant l'urgence des besoins, invite les députés à hâter leurs délibérations. Le 9 prairial, c'est à nouveau sur le rapport du ministre et pièces à l'appui que le Directoire renouvelle son invitation, avec succès cette fois puisque trois semaines plus tard, Challan présente un nouveau projet révisé au nom de la commission fluviale (39). La ténacité du ministre est en l'occurrence à mettre en relation avec le grand projet d'amélioration des communications fluviales, dont la septième division du ministère, dite « de la navigation intérieure », organisée par ses p. 321 .

(36) Id. Table chronologique, p. 327. Procès verbal du Conseil des Cinq-Cents, 14 fructidor an V, chap. 12.

(37) Sur ces deux exemples, cf. Dominique MARGaIRAz, Du lieu commun d l'esprit public..., op. cit,

(38) BOREL-VERNIÈRE, Rapport et projet de résolution présentés au nom d'une commission spéciale sur la navigation fluviale séance du 28 frimaire an VII, Imprimerie de la République, an VII. Message du 29 pluviôse an VII : Procès-verbal du Conseil des Cinq-Cents, 2 ventôse an VII ; Registre des délibérations du Directoire exécutif, A.N., AF III*133.

(39) Message du 9 prairial an VII : Procès-verbal du Conseil des Cinq-Cents, 13 prairial an VII. Registre des délibérations du Directoire exécutif, A.N., AF III* 135. Challan, Rapport et projet de résolution présenté au nom d'une commission spéciale... sur la navigation intérieure, le 27 prairial an VII, Imprimerie de la Republique, an VII. 
soins, s'occupe alors activement, et dont la réussite est suspendue aux perspectives de financement. Mais, d'une façon générale, il est difficile d'apprécier dans quelle mesure ses initiatives sont, ou non, coordonnées avec les stratégies développées par certains représentants au sein des assemblées. La chose est d'autant moins impossible qu'il compte des relations de très longue date parmi les députés, en particulier ceux des Vosges. Mais ceux-ci ne lui sont pas forcément proches et ne partagent pas nécessairement ses vues, pas au point en tout cas de jouer les poissons pilotes pour son compte au sein de l'une ou l'autre assemblée. Au vu de la documentation, la chose est à peu près indécidable. La réponse serait au demeurant de faible profit pour le présent propos, apte seulement à nous renseigner sur d'éventuelles variantes stratégiques, sans remettre en cause le fond et le sens de la démarche du ministre.

Quel que soit le terrain sur lequel elles se déploient, les initiatives de François de Neufchâteau, on le voit, concouraient à conférer à son ministère une visibilité peu en accord avec l'effacement de la fonction ministérielle souhaité par les constituants de l'an III. Chacune d'entre elles tendait à abaisser les frontières rigides édifiées par ceux-ci entre les différentes fonctions, à l'intérieur de la sphère de l'Exécutif ou entre celle-ci et la sphère du Législatif. En exploitant jusqu'aux limites constitutionnellement admises les différentes ressources à sa disposition, le ministre a sans relâche tenté d'orienter ou d'infléchir l'action gouvernementale comme celle du législateur, en leur procurant les connaissances et les données que seuls ses bureaux se trouvaient en mesure de réunir, et même les propositions qu'il estimait conformes à l'intérêt général et indispensables à une prise de décision éclairée. L'originalité de la démarche vient pour partie de ce qu'elle s'inscrit dans la continuité des exigences et des attentes exprimées par les constituants en matière d'exécution des lois, une prompte et rigoureuse application exigeant à ses yeux la production, constamment actualisée, de comptes de résultats dont l'analyse autorise leur amélioration, au prix d'aménagements éventuels dont il ne possédait pas la maîtrise. Le partage entre travail administratif et travail gouvernemental se trouve remis en cause, et, au-delà, l'approche met l'accent sur la nécessaire participation de l'Exécutif au processus d'élaboration des lois, soit qu'il puisse en assumer l'initiative sous la forme de projets, soit qu'il puisse y collaborer par la production d'informations et l'exposé de son point de vue. L'originalité de la démarche procède aussi de la façon dont François de Neufchâteau l'a assumée, tout à fait ouvertement et en son nom propre, sans tenter par ailleurs d'en extraire les principes d'une théorie constitutionnelle. En choisissant d'intégrer au recueil de ses circulaires les arrêtés et les messages qui témoignaient de ses efforts pour sortir du carcan contraignant que la Constitution imposait à sa fonction, en réintégrant dans le 
corpus de ses actes administratifs une parole à la première personne au moyen d'un savant appareil critique, en décidant enfin de publier ce recueil et de le porter ainsi à la connaissance du public, il entendait assumer pleinement sa responsabilité de ministre, non seulement auprès du Directoire, mais aussi devant la représentation nationale et, au-delà, devant la Nation toute entière. Si, par sa pratique, François de Neufchâteau avait anticipé les révisions consulaires, il esquissait aussi une figure de la responsabilité ministérielle que Benjamin Constant place au cœur de sa réflexion politique quelques quinze ans plus tard (40).

Dominique MARGAIRAZ

Université Paris I Panthéon-Sorbonne Institut d'histoire économique et sociale

17 , rue de la Sorbonne 75231 Paris Cedex 05

dominique.margairaz@univ-paris1.fr 